\title{
An Assessment of the Origin of Contrast in Off-Axis Electron Holographic Imaging of $\mathrm{BaTiO}_{3}$ Ferroelectric Domains
}

\author{
Vasiliki Tileli ${ }^{1}$, David Cooper ${ }^{2}$ and Rafal E. Dunin-Borkowski ${ }^{3}$ \\ ${ }^{1}$ Department of Materials, Imperial College, Exhibition Road, SW7 2AZ London, UK \\ ${ }^{2}$ CEA, LETI France MINATEC Campus, 17 rue des Martyrs, 38054 Grenoble Cedex 9, France \\ ${ }^{3}$ Ernst Ruska-Centre for Microscopy and Spectroscopy with Electrons and Peter Grünberg \\ Institute, Research Centre Jülich, D-52425 Jülich, Germany
}

Off-axis electron holography is a direct phase-contrast transmission electron microscopy (TEM) technique providing a record of the whole image, i.e. both the amplitude and the phase of the complex electron wave function are simultaneously recorded. Variations in electrostatic (and magnetic) fields affect primarily the phase of the electron wave function, thus in principle, electron holography is the most effective technique that can map directly the electrostatic (and magnetic) field distributions both at a nanometer and sub-nanometer scale [1]. However, a number of issues make the interpretation of electron holography results challenging. Here, we discuss the implications of electron holography applied to the characterization of electrostatic potentials in ferroelectric materials.

Ferroelectrics are materials that possess a spontaneous electric polarization, which can be reversed by applying a suitable external electric field. Their polarization, and thus their exceptional properties, is attributed to the dipoles formed by displacements of charges ions inside the crystal unit cell . $\mathrm{BaTiO}_{3}$ is a perovskite crystal with a Curie temperature of $120^{\circ} \mathrm{C}$ that exhibits four different distinct phases. The changes in phase (first order transitions) correspond to discontinuities in the direction of spontaneous polarization. At room temperature, barium titanate is in the tetragonal phase and its polarization is oriented in the $<100>$ direction [2].

Figs 1 and 2 depict medium resolution phase and amplitude images (as extracted from off-axis electron holograms) of $\mathrm{BaTiO}_{3}(100)$ domains in room temperature and above the Curie temperature respectively. The images were acquired at $300 \mathrm{kV}$ in Lorentz mode. In room temperature, Fig. 1, the domains are clearly visible in the phase image but contrast appears partly in the amplitude image as well. Above the Curie temperature, where $\mathrm{BaTiO}_{3}$ ceases to be ferroelectric, Fig 2, the phase exhibits a gradual decrease across the sample, whereas the amplitude remains almost constant, indicative of the thickness uniformity of the specimen.

Few studies have emerged recently focusing on off-axis electron holography on $\mathrm{BaTiO}_{3}$ domains $[3,4]$. Their attempt is to evaluate the phase changes inherent to the ferroelectric nature of the material. However, many factors influence such measurements and consequently give rise to imaging artifacts. For instance, the fringing fields extending above and below the specimen surface (originating from amorphization of the surfaces of the crystalline specimen during its TEM preparation process) impose complications on the interpretation. In addition, the effect of dynamical diffraction on the measured phase shift is difficult to be minimized for ferroelectric crystals. The dramatic influence of specicem tilt/orientation is seen in Figure 1. Phase shifts of the electron wave can occur inhomogeneously across the specimen due to diffraction from domain wall structure for example. The passage of high-energy electrons through the ferroelectric oxide specimen will inevitably cause charging artifacts. This effect induces the substantial phase decay as depicted in Figure 2c. Thus, residual charged sites in the material will cause measured phase shifts to deviate from the undisturbed ferroelectric domain structure. 
All factors that can lead to a substantially alteration of the projected distribution of charges will be discussed and routes for minimization of their contribution towards imaging artifacts will be proposed.

\section{References:}

[1] R.E. Dunin-Borkowski et al., "Encyclopedia of Nanoscience and Nanotechnology", American Scientific Publishers: Stevenson Ranch, CA, Vol. 3 (2004) 41.

[2] P. W. Forsbergh, Phys. Rev. 76 (1949), p.1187.

[3] T. Matsumoto et al., Appl. Phys. Lett. 92 (2008), p.072902.

[4] M. J. Polking, et al., Nat. Mat. 11 (2012), p.700.

[5] The research leading to these results has received funding from the People Programme (Marie Curie Actions) of the European Union's Seventh Framework Programme (FP7/2007-2013) under REA grant agreement $\mathrm{n}^{\circ}[301838]$.
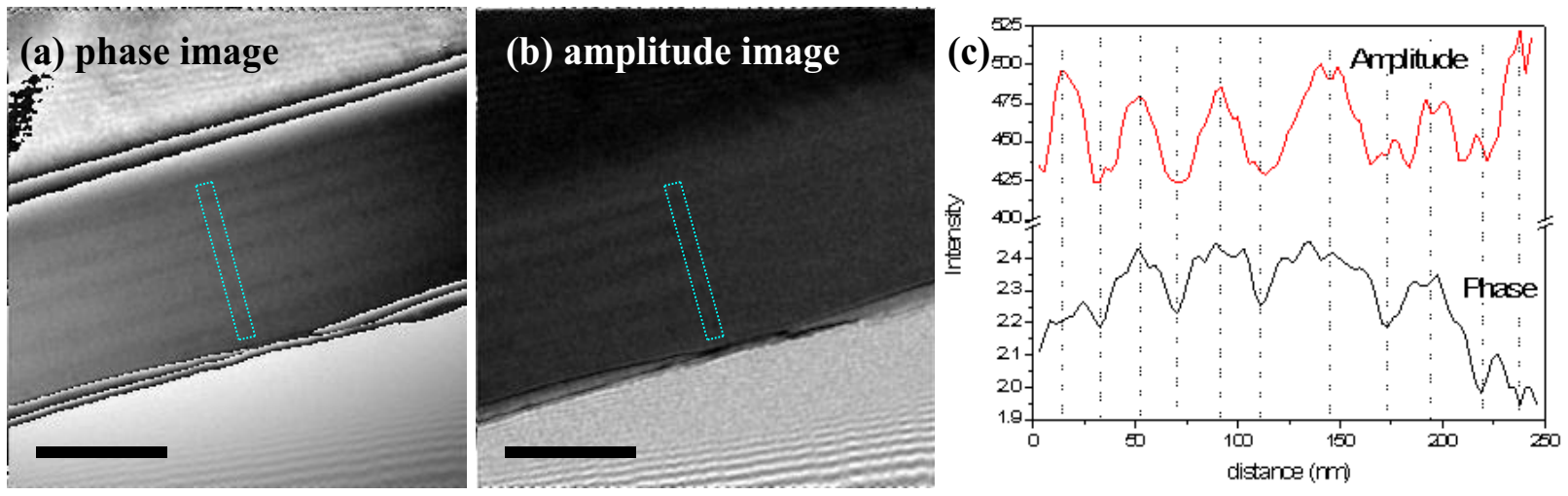

Figure 1. (a) Phase and (b) amplitude image of single crystal $\mathrm{BaTiO}_{3}(001)$ taken at room temperature. The specimen was tilted away from the zone axis for acquisition of the holograms. The dark lines across the structure in the phase image correspond to contrast related to ferroelectric domains. Some contrast is also evident at the amplitude image however it is more diffuse and it diminishes towards the mid of the irradiated area. The profiles from the mid regions shown in (a) and (b) are shown in (c). Contrast in both images seems to be related.
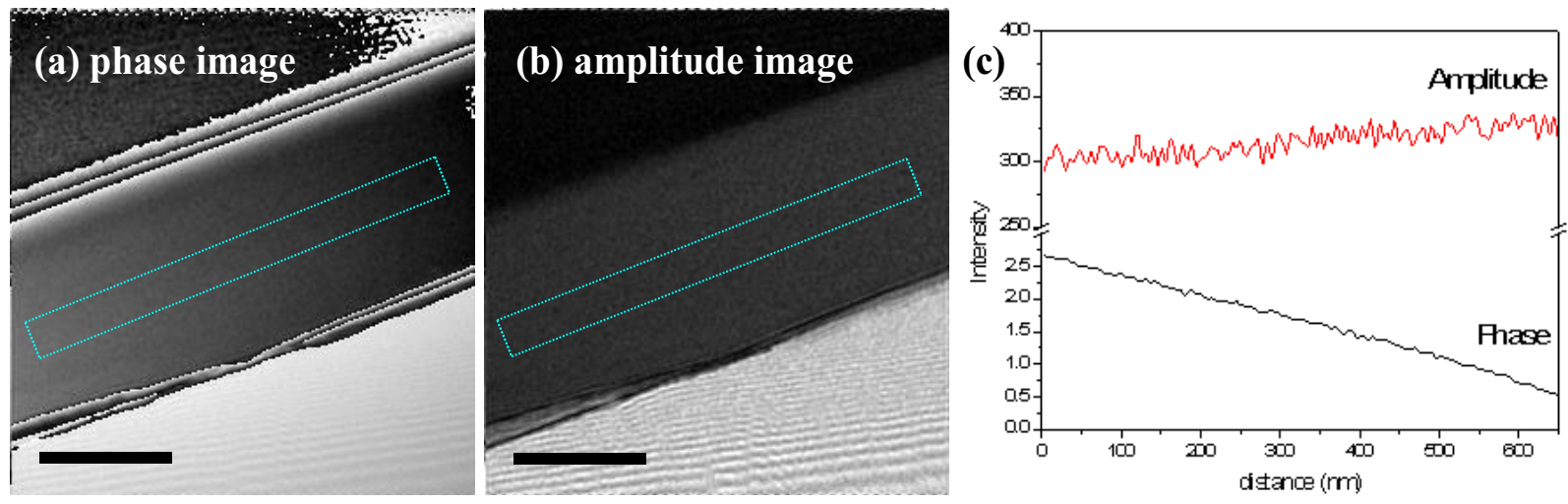

Figure 2. (a) Phase and (b) amplitude image of single crystal $\mathrm{BaTiO}_{3}(001)$ taken above the Curie temperature (at $150^{\circ} \mathrm{C}$ ). Phase decay as shown in (c) is related to specimen charging. Small variation in amplitude contrast is attributed to minor thickness variation across the specimen. 